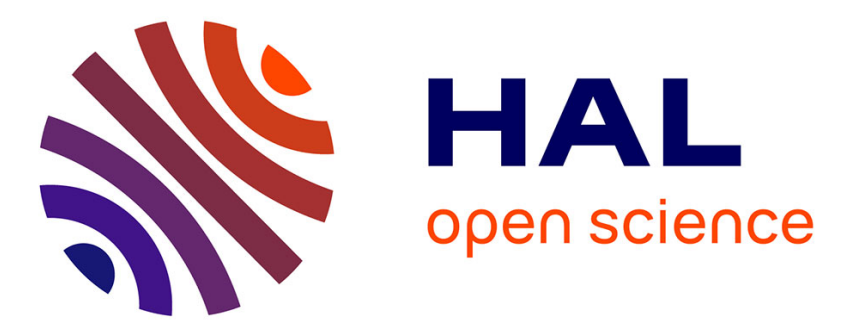

\title{
Extension of EWF Threat Model and Associated SQM
}

Olivier Julien, Ikhlas Selmi, Jean-Baptiste Pagot, Francisco

Amarillo-Fernandez

\section{To cite this version:}

Olivier Julien, Ikhlas Selmi, Jean-Baptiste Pagot, Francisco Amarillo-Fernandez. Extension of EWF Threat Model and Associated SQM. ITM 2017, ION International Technical Meeting, ION, Jan 2017, Monterey, United States. pp.492-507, 10.33012/2017.14873 . hal-01521943

\section{HAL Id: hal-01521943 \\ https://hal-enac.archives-ouvertes.fr/hal-01521943}

Submitted on 8 Oct 2020

HAL is a multi-disciplinary open access archive for the deposit and dissemination of scientific research documents, whether they are published or not. The documents may come from teaching and research institutions in France or abroad, or from public or private research centers.
L'archive ouverte pluridisciplinaire HAL, est destinée au dépôt et à la diffusion de documents scientifiques de niveau recherche, publiés ou non, émanant des établissements d'enseignement et de recherche français ou étrangers, des laboratoires publics ou privés. 


\title{
Extension of EWF Threat Model and Associated SQM
}

\author{
Olivier Julien, Ikhlas Selmi, Jean-Baptiste Pagot, ENAC, Toulouse, France \\ Jaron Samson, Francisco Amarillo Fernandez, European Space Agency
}

\section{BIOGRAPHIES}

Dr. Olivier Julien is the head of the Signal Processing and Navigation (SIGNAV) research group of the TELECOM laboratory of ENAC, in Toulouse, France. He received his engineer degree in 2001 in digital communications from ENAC and his PhD in 2005 from the Department of Geomatics Engineering of the University of Calgary, Canada. His research interests are turned towards the use of satellite-based navigation systems for safe navigation.

Dr. Ikhlas Selmi is currently part of the SIGnal processing and NAVigation (SIGNAV) research group of Telecom lab at ENAC (Ecole Nationale de l'Aviation Civile). She received a Master of Science in Telecommunication and Signal Processing from ESIEE (Ecole Supérieure d'ingénieur en Electronique et Electrothechnique) and a Master of Research in High Frequency Communication System from University Paris-Est, Marne la Vallée. She obtained her Ph.D. in 2013 from Telecom SudParis. Her Ph.D thesis dealt with optimizing indoor positioning system based on GNSS transmitters, and more specifically, on mitigating interference between indoor and outdoor signals and simplifying the system's architecture. Then she was a postdoctoral fellow at University of Littoral-Côte-d'Opale (ULCO) where she worked on designing an underwater positioning system for low depth environment. She is currently working on GNSS Signal distortion generated by the satellite payload.

Jean-Baptiste Pagot is a PhD candidate in the Department of Signal Processing and Navigation (SIGNAV) at the ENAC (Ecole Nationale de l'Aviation Civile) in Toulouse, France. He received his master degree as an Electronics and Telecommunication engineer in 2013 from the ENAC. He is currently working on GNSS Signal Distortions.

Jaron Samson has been working as a System Engineer at ESA's EGNOS Project Office in Toulouse (France) since 2012. Prior to 2012, he worked as a Navigation Engineer at ESA's Technical Centre (ESTEC), supporting Galileo, EGNOS and the GNSS Evolutions Program. Jaron holds an M.Sc-degree in Physical and Mathematical Geodesy from Delft University of Technology (The Netherlands).

Francisco Amarillo-Fernández received his Master's Degree in Telecommunication by the Polytechnic University of Madrid (UPM) Spain in 1997, and his Master's Degree in Surveying Engineering by the UPM in 1992. He has been working for the ESA Navigation Directorate since 2001 and has participated/led in numerous research activities in the GNSS field since 1997.

\begin{abstract}
The consequences of GPS L1 C/A signal distortions due to payload failure on a civil aviation GPS user has received a lot of attention in the late 1990's and early 2000 following the occurrence of a first such event in 1993. The result of the corresponding work led to the characterization and modeling of such a threat, known as Threat Model (TM), and the development of means to mitigate this threat in SBAS and GBAS, known as Signal Quality Monitoring (SQM).

The proposed TM, endorsed by International Civil Aviation Organization (ICAO), is adapted to the GPS L1 C/A signal and derived from the observations of the 1993 event as well as other considerations. However, with the evolution of the payloads over the years, the multiplication of GNSSs, and the multiplication of modulations used by GNSS signals, the adaptation of this TM to other signals and systems might not be straight-forward: the a priori identification of the effect of a payload failure on the transmitted signal is a very complicated task.

The objective of this paper is thus to investigate another TM, referred to as generic TM, that could cover a large threat space and that could be adapted to other signals. This generic TM could be for instance used by future Dual-Frequency Multi-Constellation SBAS to protect the use of new GNSS signals. The article thus investigates such generic TM in the specific case of Galileo E1C and its impact on an associated SQM.
\end{abstract}

\section{INTRODUCTION}

In 1993, the first important GNSS signal distortion due to a payload failure was observed. This type of distorted signal could be broadcast for a long time without the system raising an alert while it could generate large measurement errors for both standalone and differential users, thus their nickname Evil WaveForm (EWF). This raised two important questions among the user 
communities, and more particularly the civil aviation community: What kind of signal distortions due to payload failure could affect a GNSS signal in a hazardous way? How is it possible to protect a user against this threat? Civil aviation then dedicated efforts to characterize this threat and put specific protection mechanisms in place for SBAS and GBAS users. Three signal distortion types (due to payload failure), also referred as Threat Models (TM), leading to dangerous correlation function distortions were adopted in 2001 by ICAO for GPS L1 C/A [ICAO, 2006]. These TM were defined based on the satellite payload knowledge and the observation of the 1993 event:

- TM-A which is associated with a failure in the navigation data unit (NDU), the digital part of a satellite. It consists of the normal C/A code signal except that all the positive chips have a falling edge that leads or lags relative to the correct end-time for that chip $(\Delta)$.

- TM-B which introduces amplitude modulation and models degradations in the analog section of a satellite. More specifically, it consists of the output from a second order system when the nominal C/A code baseband signal is the input. Two parameters are defined for this threat model: the damping factor $\sigma$ and the ringing frequency $f_{d}$.

- $\quad$ TM-C which is a combination of the two first failures.

This definition of these TM was associated with the need to have a monitoring mechanism in order to ensure that the occurrence of a signal distortion according to the defined TM would not affect adversely an airborne user: either the distortion is detected and the user warned in due time, or the distortion is not detected, but in this case it should not create an integrity issue. This monitoring mechanism is referred to as Signal Quality Monitoring (SQM) and is currently implemented in SBAS and GBAS ground infrastructures (in different ways).

It is well known that the current ICAO TM for GPS L1 C/A is only a model of the EWF phenomenon based on the observation of a limited number of events and that with the evolution of payload technologies and the transmission of new GNSS signals using different modulations, new EWF TM might need to be defined for new GNSS signals. This issue was already studied in [Phelts, et al, 2006; Fontanella et al, 2014, Pagot et al, 2015] where the investigation was turned towards an adaptation of the same types of TM (A, B and C) as the accepted GPS L1 C/A ICAO TM on new signals, but revising the range of parameters the distortions could take.

However, this approach might restrict the TM family to a set of distortions that do not encompass all potential distortions created by current payloads and signals. As a consequence, the present article aims at investigating a wider TM definition that would allow protecting the airborne user against a wider range of potential signal distortions. Obviously, assuming a wider TM also means that the SQM has to be adapted to this TM, and more likely more protective. Consequently, the second goal of this article is to look at the performance of a baseline SQM that would be adapted to the new TM. The core of the article will be oriented towards the case of Galileo E1 OS signal. Note that this investigation is also done in the frame of a future Dual Frequency MultiConstellation (DFMC) SBAS system.

This article is thus organized as follows:

- The first part of the paper will provide the foundations that are used to define the new TM.

- The second part of the paper will discuss how this new TM could be reduced (in terms of parameter range) in order to facilitate the design of the SQM and allow for an easier testing.

- $\quad$ The third part of the paper will describe the considerations used for the proposed baseline SQM.

- The fourth part will describe the testing scenarios

- The fifth part will discuss results obtained with Galileo E1 OS.

- For comparison purpose, the sixth part will look at the impact of the generic TM on GPS L1 C/A

- Finally a conclusion on the presented results and the way forward will be provided.

\section{DEFINITION OF THE PROPOSED GENERIC THREAT MODEL}

\section{Presentation of the Generic Threat Model and its Key Parameters}

The proposed generic TM consists in defining the unfiltered correlation function distortion $R_{d}$ due to a payload malfunction as the finite sum of cosine components according to the following model:

$$
\begin{aligned}
R_{d}(\tau)= & \sum_{k=0}^{N} d_{k} \cos \left(2 \pi f_{k} \tau+\varphi_{k}\right) \\
& \text { with } \sum_{k=0}^{N}\left|d_{k}\right|<D
\end{aligned}
$$

where the key parameters of the model are:

- $\quad d_{k}$ is the amplitude of the $k^{\text {th }}$ cosine component. 
- $\quad f_{k}$ is the frequency of the $k^{\text {th }}$ cosine component.

- $\quad \varphi_{k}$ is the phase of the $k-t h$ cosine component.

- $\quad N$ is the number of components constitutive of the distortion.

- $\quad D$ is fixed to a finite value

The idea is to try to define a generic model based on the Fourier decomposition of any distortion. As a consequence, there are different ways to express this model (with complex exponential terms, with real or complex amplitude terms, etc...). All these notations being equivalent, the one above will be used in this document. Although the proposed generic TM is expressed in the correlation domain, it can also be translated in the time domain.

The limitation of $D$ to a finite value is meant at making this model realistic as the distortion coming from the payload will have a finite power. This generic TM differs from the current ICAO TM for GPS L1 C/A in different ways:

- The GPS L1 C/A TM makes a priori assumptions on the shape of the distortions, while the generic model assumes that the distortion can have any shape.

- The threat space of the proposed generic TM is much larger than that of the ICAO TM. In fact, the threat space of the generic TM is for now infinite, which could significantly complicate the testing of a system considering the generic TM as its TM.

\section{A Priori Range of Parameters of the Generic TM}

\section{Range of the Frequency Components}

A component with $f_{k}=0$ is equivalent to a constant amplitude change of the whole distorted correlation function. After the correlation function normalization, this amplitude change would be totally absorbed. This is why the zero frequency component is not taken into account in the model.

The distortion can be composed of high frequency components. Nevertheless, the important criteria to take into account in the evaluation of the worst different tracking bias is the impact of a filtered (by the payload and receiver RF front-end) distortion added to a filtered correlation function. Consequently, it can be assumed that distortions' high frequencies can be removed from the TM because they will have a negligible impact after applying the RF filter. It is well known that the SBAS reference and airborne civil aviation receivers have a double-sided RF front-end filter that will not exceed $24 \mathrm{MHz}$ ( 3 -dB). It is thus reasonable to only consider frequency components of the distortion that are slightly in excess of $12 \mathrm{MHz}$. In order to take into account a potential slower attenuation of the filter amplitude response with frequency, it was decided to take a margin and to set a maximum frequency to any distortion component equal to $30 \mathrm{MHz}$.

\section{$\underline{\text { Range of } D}$}

One way of limiting the value of $D$ in the generic TM is to assess the maximum possible distortion power that could be created by the payload. This data not being readily available, it is possible to assess a rough value of $D$ by looking at its value if we represent the current GPS L1 C/A ICAO model as a member of the generic TM. The analysis performance showed that the maximum value of $D$ for the current GPS L1 C/A ICAO model is 0.13 . Such a value gives an interesting order of idea for reasonably bounding $D$, although this is not based on direct payload information.

\section{Conclusions}

Based on the above analysis and the will to include some key parameters of the current ICAO TM for GPS L1 C/A in the proposed generic TM (in particular the power of the distortion), the initial range of values of the key parameters of the generic TM, also known as Threat Space (TS) is summarized in Table 1.

Table 1. Threat Space associated to the proposed generic TM

\begin{tabular}{|c|c|c|c|}
\hline $\boldsymbol{N}$ & $\boldsymbol{D}$ & $\boldsymbol{\varphi}_{\boldsymbol{k}}(\mathrm{rad})$ & $\boldsymbol{f}_{\boldsymbol{k}}(\mathrm{MHz})$ \\
\hline$[0 ;+\infty]$ & {$[0 ;$ at least 0.13$]$} & {$[0 ; 2 \pi]$} & ] $0 ; 30]$ \\
\hline
\end{tabular}

The generic TM proposed above is clearly very large and it is important to make an effort to reduce it so that it can be workable. By doing so, the model will lose some of its original characteristics, which is to represent almost any type of distortion, but this is essentially for practical reasons. One way to reduce the generic TM threat space is to remove the ranges of parameters that would make the corresponding distortions easily detected by a baseline SQM. In this case, it is important to define a baseline 
SQM that could be representative of an SQM adapted to a future DFMC SBAS reference receiver. This is done in the next section.

\section{PROPOSED BASELINE SQM AND PERFORMANCE ASSESSMENT}

\section{Baseline SQM and Required Performance}

The SQM required performance can be derived from ICAO requirements [SARPS, 2006] or from other performance data that are available, such as [Phelts et al, 2013]. It can then be derived to the reference receiver-level based on the choice of a SBAS SQM architecture. Finally, it can be derived to the EWF detector level using assumptions on the type of detectors used, and the correlation between the detectors used. In the present case, a decentralized SBAS SQM architecture is assumed, where the EWF detection is performed at each SBAS reference station level, with a decision on the presence of an EWF at system level taken by the Central Processing Facility (CPF) based on majority voting of at least 4 reference stations. Based on these assumptions, and on the fact that the SBAS reference receiver and airborne receivers is expected to fulfill other requirements expressed in [ESA, 2016], Table 2 provides the key baseline reference receiver configuration. Note that Table 2 also provides the considered airborne receiver range of configurations, also known as User Design Space (UDS) [ESA, 2016].

Table 2 - Receiver Baseline Configuration

\begin{tabular}{|c|c|c|c|c|}
\hline Signal & $\begin{array}{ll}\text { SBAS } & \text { SQM } \\
\text { architecture }\end{array}$ & $\begin{array}{l}\text { Worst } \\
\text { Case C/No }\end{array}$ & $\begin{array}{l}\text { Reference station } \\
\text { receiver constraints }\end{array}$ & $\begin{array}{l}\text { Airborne receiver constraints and } \\
\text { User Design Space }\end{array}$ \\
\hline Galileo E1 OS & $\begin{array}{l}\text { at least } 4 \text { Reference } \\
\text { Stations (RS) } \\
\text { observing a given } \\
\text { satellite. Decision of } \\
\text { EWF based on at } \\
\text { least } 3 \text { RS with } \\
\text { positive detection }\end{array}$ & $30 \mathrm{~dB}-\mathrm{Hz}$ & $\begin{array}{l}\text { - } \text { BOC }(1,1) \text { receiver } \\
\text { - } \text { BW2 of } 24 \mathrm{MHz}\left(6^{\text {th }}-\right. \\
\text { order Butterworth }) \\
\text { - } \text { EML discriminator } \\
\text { - E-L spacing of } 0.1 \text { chip }\end{array}$ & $\begin{array}{l}\text { - } \text { BOC(1,1) receiver } \\
\text { - } \text { BW2 within [12-24] } \mathrm{MHz} \text { (4 } \\
\text { different filters) } \\
\text { - EML discriminator } \\
\text { - E-L spacing within [0.08-0.12] } \\
\text { chip }\end{array}$ \\
\hline
\end{tabular}

The proposed baseline SQM has to be adapted to the reference receiver configuration. In this article, a reference receiver configuration significantly more complex than the one currently used in WAAS or EGNOS is assumed for mainly two reasons: receiver technology has significantly evolved since the design of the current SQM, and the generic TM is anticipated to require a stronger SQM. This reference receiver complexity could anyway be reduced a posteriori if it is assessed that the SQM does not need such a complex receiver.

The baseline SQM is thus based on the use of 51 correlators mapping the correlation function from -0.25 to 0.25 chips with a step of 0.01 chip between correlators. as it is expected that the proposed generic TM, due to its significantly greater UDS, would require a quite complex SQM. Only 3 types of metrics are considered for the SQM: simple ratio, difference ratio and sum ratio metrics all normalized by the prompt correlator output, as presented in [Pagot et al, 2016]. The consideration of these 3 types of metrics coupled with the availability of 51 correlators provides a total of 100 usable metrics that can be used to detect the presence of an EWF. Each metric is associated to a distortion detector that corresponds to the difference between the measured metric value and the expected metric value based on the expected nominal correlation function. A distortion detector will then flag the presence of an EWF when its value is greater than a predefined threshold. This threshold is based on a required false alarm probability and the knowledge of the detector distribution, which will be assumed Gaussian in the rest of this document. It is to be noted that a detection is declared at the reference receiver level when at least one of the distortion detectors of the baseline SQM flags an EWF.

The occurrence of an EWF entailing a differential measurement error greater than the Maximum ERRor (MERR) of 1.55m at on Galileo E1 is here considered as a threat. The choice of $1.55 \mathrm{~m}$ is derived from the MERR value of $3.5 \mathrm{~m}$ often found for an SBAS L1 mapped to the case of a DFMC SBAS that uses ionosphere-free measurements [Phelts et al, 2013]. Note that more advanced choices for the MERR have been discussed in [Shively, 1999, Schloss et al, 2002, Rife and Phelts, 2008; Phelts et al, 2013] and will be taken into in later studies.

The required false alarm and missed detection probability per distortion detector to protect the user was derived from the global ICAO SQM requirement, the assumed SBAS architecture and considering the different metrics correlation. 
It is finally assumed that the metrics are smoothed over 50-sec as suggested in [Phelts et al, 2013] to reduce the noise present at the metric (and thus detector) level. The effect of this smoothing, taking into account the presence of a worst case multipath scenario, can be represented as a reduction of the raw metric standard deviation by a factor of 1.5 [Pagot et al, 2016].

Table 3 summarizes all the key elements of the SQM configurations.

Table 3 - SQM per Reference Station Baseline Configuration

\begin{tabular}{|c|c|c|c|c|c|}
\hline & $\begin{array}{l}\text { Location } \\
\text { correlators }\end{array}$ & Metrics & $\begin{array}{l}\text { Metric } \\
\text { Smoothing }\end{array}$ & MERR & $\begin{array}{lr}\text { False alarm } & \text { and } \\
\text { missed } & \text { detection } \\
\text { probabilities }\end{array}$ \\
\hline Galileo E1 OS & $\begin{array}{c}51 \text { correlator } \\
\text { outputs at } \\
-0.25: 0.01: 0.25 \\
\text { chips }\end{array}$ & $\begin{array}{l}\text { - Simple ratios } \\
\text { - Difference } \\
\text { ratios } \\
\text { - Sum ratios }\end{array}$ & $\begin{array}{c}50-\mathrm{s} \\
\text { corresponding } \\
\text { to a reduction of } \\
\text { the std by a } \\
\text { factor of } 1.5\end{array}$ & $1.55 \mathrm{~m}$ & $\begin{array}{c}-\mathrm{P}_{\text {fa }} \text { per metric per test } \\
\text { of } 10^{-4} \\
-\mathrm{P}_{\text {md }} \text { per metric per } \\
\text { test of } 1.2 \cdot 10^{-3}\end{array}$ \\
\hline
\end{tabular}

\section{Baseline SQM Performance Assessment}

For a given distortion detector, the requirements can be directly related to the notion of minimum detectable metric bias. This minimum detectable metric bias is also referred to as Minimum Detectable Error (MDE) or Minimum Detectable Ratio (MDR). For the sake of notation, we will only use the notation MDE in the rest of this article.

It can be shown that for long integration duration, the noise at the output of the unsmoothed metrics can be considered Gaussian. This means that the distortion detector can be assumed to be a centered Gaussian random variable in the absence of EWF, and a non-centered Gaussian random variable in the presence of an EWF (steady-state case). As a consequence, the MDE/MDR can be expressed for a given detector as:

where

$$
M D E=\left(K_{m d}+K_{f f d}\right) \sigma_{m e t r l c} \widetilde{e}^{l}
$$

- $\quad K_{f f d}$ is a typical fault-free detection multiplier representing a given false detection probability;

- $K_{m d}$ is a typical missed detection multiplier representing a given missed detection probability;

- $\sigma_{\text {metrlc }}$ is the standard deviation of the distortion detector, which is equal to the standard deviation of the unsmoothed metric

With the false alarm and missed detection probabilities given in Table 3, the MDE for each metric can be computed as :

$$
M D E^{i}=7.13 \times \sigma_{m e t r i c} \widetilde{m}^{2}
$$

From this expression, it is easy to understand that an important element to improve the EWF detection is to have a standard deviation of the metric as small as possible. It is thus important to also accurately model $\sigma_{m e t r i c}$. This can be quite tricky as the effect of the metric smoothing (if any) has to be taken into account. As provided in Table 3, the baseline SQM assumes a smoothing corresponding to a worst case reduction of the metric standard deviation by a factor of 1.5 (assuming the presence of correlated multipath). As a consequence, the computation of $M D E^{i}$ can be revisited as:

$$
M D E^{i}=4.75 \times \sigma_{\text {metric }}{ }^{\prime} \text {,unsmoothed }
$$

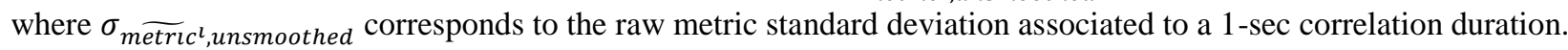

Note that the possibility to use a centralized SBAS architecture that would be based on the reference stations transmitting their correlator outputs or metrics to the CPF has also been analyzed. In this case, it can be shown that the MDE would be (taking into account 4 reference stations providing their inputs):

$$
M D E^{i}=3.55 \times \sigma_{\text {metric }} \text {, unsmoothed }
$$

This corresponds to a reduction of the MDE by $25 \%$ compared to the considered decentralized architecture, and thus an improvement by as much of the detectability of the EWF. The considered decentralized can thus be considered as a conservative case in terms of performance (although the centralized architecture also has its drawbacks with some fine-tuning to be done).

[Pagot et al, 2016] provides a model of $\sigma_{\text {metric }}$, unsmoothed as a function of the metric assuming thermal noise only. Note that

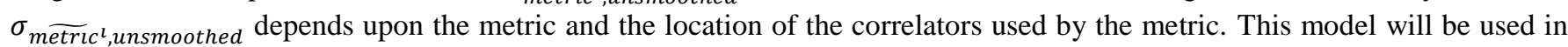
the rest of this document. 
As a consequence, in a given configuration, the performance of the SQM can be assessed by looking if a given distortion makes at least one of the distortion detectors greater than its corresponding $M D E^{i}$. If this happens, it means that the corresponding distortion should be detected by the reference station according to the ICAO requirements. This can then be played against the whole generic TM to distinguish between distortions that can be detected according to the requirements, and the ones that cannot. Note that this approach is only considering a steady-state behavior and ignores the effect of the different filters (airborne measurement smoothing, metric smoothing) and the Time-to-Alert (TTA) requirements. It thus only provides a first idea.

\section{SQM TEST METHODOLOGY}

\section{Description of the test methodology}

Because the proposed generic TM is for now consisting of an infinite threat space, it has been decided to first assess the impact of the new generic TM on the baseline SQM through heavy simulations. This solution is only a first step meant at better understanding the capability of the baseline SQM against the generic TM since:

- the simulations would require an infinite number of iterations to cover all possible cases. This is even more pronounced with the present generic TM which requires a huge number of simulations (at least several millions) to start converging (any number of components with any frequency and any phase and any amplitude).

- The simulation are associated with a specific SQM and the results obtained with a specific SQM might be different with another SQM.

In any case, the idea of these tests is to try to gather a general knowledge of the sensitivity of the different parameters that could lead to a drastic reduction of the generic TM parameter space. To do so, the simulation mechanism has to be specified. The simulation set up is represented in Figure 1. The algorithm is the following:

- Generate an ideal correlation function.

- Add a distortion based on the generic TM. In the frame of the simulations, the number of components of the distortion has been limited to a maximum of 10 in order to limit the number of distortions to generate to reach stable results. This can appear small, but it was assessed to still allow for representative results. Each TM component is then drawn according to:

- A random initial phase (uniform distribution)

- A random frequency smaller than $f_{\max }=30 \mathrm{MHz}$ (uniform distribution)

- A random amplitude for each component with a constraint on the value of $D$. Based on the analysis of the power of the distortion allowed by the GPS L1 C/A ICAO TM, it was decided to use a value of $D$ equal to 0.13 .

- The distorted correlation function is then:

- passed through one equivalent RF front-end filter representative of the reference receiver

- all metrics of the SQM are computed and compared against their respective MDE

- The same distorted correlation function is also:

○ passed in parallel through a bank of equivalent RF front-end filters representative of different airborne RF filter technologies and bandwidth

○ For each filter output of the bank, the differential tracking bias between the reference and the airborne receiver is assessed based on a given correlator spacing for the reference receiver and different user correlator spacing.

- The worst differential tracking bias across the different RF front-end filters is kept in memory with its parameter (amplitudes, frequencies, chip spacing, filter type and bandwidth) 


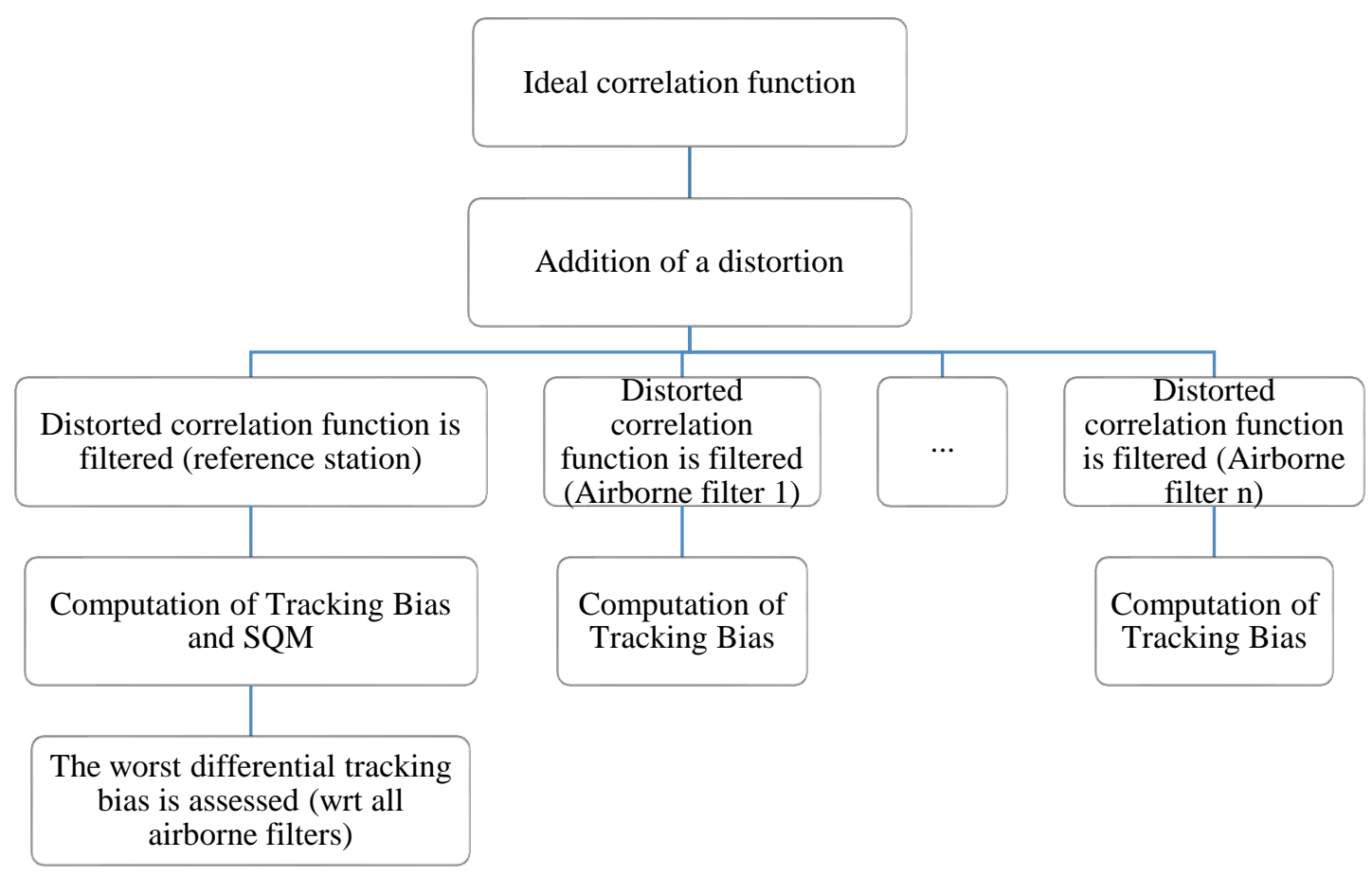

Figure 1. Different Tracking Bias Estimation Simulation Set Up

The different airborne/reference user configurations used during the simulations are provided in Table 4 (they are in line with the UDS described earlier).

Table 4. Tested user and reference configurations

\begin{tabular}{|c|c|c|c|c|}
\hline \multirow{2}{*}{} & \multicolumn{2}{|c|}{$\begin{array}{c}\text { Galileo E1C signal (CBOC(6,1,1/11)) } \\
\text { and GPS L1 C/A }\end{array}$} & \multicolumn{2}{c|}{ GPS L1 C/A signal (BPSK(1)) } \\
\cline { 2 - 5 } & Reference & User & User \\
\hline $\begin{array}{c}\text { Tracking } \\
\text { technique }\end{array}$ & $\begin{array}{c}\text { E-L (BOC(1.1) } \\
\text { local replica) }\end{array}$ & $\begin{array}{c}\text { E-L (BOC(1.1) } \\
\text { local replica) }\end{array}$ & $\begin{array}{c}\text { E-L (BPSK(1) } \\
\text { local replica) }\end{array}$ & $\begin{array}{c}\text { E-L (BPSK(1) } \\
\text { local replica) }\end{array}$ \\
\hline $\begin{array}{c}\text { Correlator } \\
\text { spacing }\end{array}$ & 0.1 chip & $\begin{array}{c}0.08,0.09,0.1, \\
0.11,0.12 \text { chip }\end{array}$ & 0.1 chip & 0.1 chip \\
\hline $\begin{array}{c}\text { Pre-correlation } \\
\text { bandwidth } \\
\text { (double sided) }\end{array}$ & $24 \mathrm{MHz}$ & $\begin{array}{c}12,14,16,18,20, \\
2224 \mathrm{MHz}\end{array}$ & $24 \mathrm{MHz}$ & $12,24 \mathrm{MHz}$ \\
\hline $\begin{array}{c}\text { Equivalent } \\
\text { reception filter }\end{array}$ & $\begin{array}{c}\text { 6-th order } \\
\text { Butterworth }\end{array}$ & $\begin{array}{c}4 \text { filters are tested } \\
\text { (2) }\end{array}$ & $\begin{array}{c}\text { 6-th order } \\
\text { Butterworth }\end{array}$ & 4 filters are tested \\
\hline
\end{tabular}

\section{Visualisation of Results}

For each distortion tested, the maximum value of all the ratios between the distortion detectors $D e t^{i}$ of a reference station and its associated $M D E^{i}$ can be computed as:

$$
\overline{F O M}_{\text {max }}=\max _{i}\left(\frac{D e t^{i}}{M D E^{i}}\right)
$$

$\overline{F O M}_{\max }$ is thus representative of the metric that "sees" the most the tested distortion at a given reference station. As a consequence, values of $\overline{F O M}_{\text {max }}$ exceeding 1 will be representative of distortions that have been detected according to the station requirements (in terms of $\mathrm{P}_{\mathrm{fa}}$ and $\mathrm{P}_{\mathrm{md}}$ ). $\overline{F O M}_{\max }$ can also be referred to as the detectability of a given distortion. It is then possible to associate the value of $\overline{F O M}_{\max }$ to the corresponding worst case differential tracking bias. 
As described earlier, the value of the $M D E^{i}$ for each metric is dependent upon the standard deviation of the metric itself (assumed Gaussian), which means:

- The location of the correlator used

- $\quad \mathrm{The} \mathrm{C} / \mathrm{N}_{0}$ of the received signal at the reference station at the correlator output

- The correlation duration

- The metric smoothing process (and its associate reduction of the metric standard deviation)

The figures representing the SQM performance will thus represent for each tested distortion:

- $\quad$ on the $\mathrm{x}$-axis the value of $\overline{F O M}_{\max }$ (thus the detectability of a given distortion), and

- $\quad$ on the $y$-axis the worst value of the differential tracking bias across the UDS.

As a consequence, the figures will represent a cloud of points, each point corresponding to a distortion.

The figure will be segmented in 4 sections according to:

- the value of $\overline{F O M}_{\max }$ with respect to 1 (detection according to the ICAO requirement or not)

- worst value of the differential tracking bias (above or below the MERR of $1.55 \mathrm{~m}$ for Galileo E1 OS and GPS L1 C/A in the case of a DFMC receiver as defined in [Julien et al, 2016])

Finally, the results for the generic TM will be shown as a cloud of blue points, and it will be superimposed by a cloud of green points corresponding to the baseline SQM performance against an ICAO-like TM defined for the corresponding signal. In the case of Galileo E1C, this cloud of green points is associated to the proposed ICAO-like TM defined in [Pagot et al, 2016] for Galileo E1C.

\section{RESULTS FOR GALILEO E1C}

\section{SQM Performance for Galileo E1C}

Figure 2 represents with blue circles the worst differential tracking error in the above conditions as a function of $\overline{F O M}_{\text {max }}$ for Galileo E1C over a set of 10,000,000 distortions taken from the generic TM using 1,000,000 simulated distortions per number of components present in the generated distortion. Note that this way of generating the distortions is not completely fair as:

- For small numbers of components in the generated distortion, only a small number of random parameters are drawn and the generation of 1,000,000 distortions might allow to represent most of the possible combinations of the different parameters

- For high numbers of distortions, the above might not be true. It is thus possible that what is visualized for high values of $\mathrm{N}$ is not representative of worst case scenarios, even with 1,000,000 simulated distortions

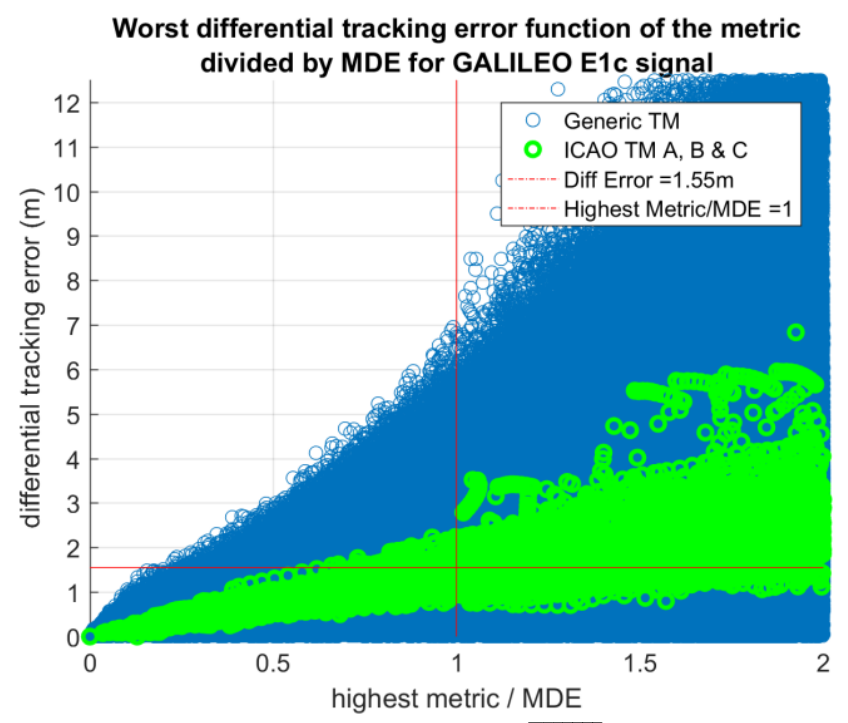

Figure 2 - Worst differential tracking error as a function of the $\overline{F O M}_{\max }$ at the Reference Station for Galileo E1C

There are many early observations that can be made from Figure 2: 
- the generic TM clearly overbounds the ICAO-like TM

- there is a large number of distortions that are undetected (according to ICAO requirements) and that are creating a differential tracking error greater than a targeted MERR of $1.55 \mathrm{~m}$. A total of 769,470 distortions are in this case

- the undetected distortions can cause large differential tracking errors. The maximum differential error for an undetected (according to ICAO requirements) distortion, also referred to as Maximum Undetected Differential Error (MUDE) is close to 8 meters, thus well above the targeted MERR of $1.55 \mathrm{~m}$.

- for a given value of detectability $\overline{F O M}_{\text {max }}$, the corresponding worst case differential tracking error can be very different

- there seems to be a fairly simple linear relation between the worst-case differential tracking error and the corresponding value of $\overline{F O M}_{\text {max }}$. This would indicate that a bounding is potentially possible.

- There are several worst case points that appear as singular points (for instance for values of $\overline{F O M}_{\text {max }}$ around and above 1). This might be caused by the fact that the number of simulated distortions, although very high, is not sufficient to be fully representative of all the types of distortions allowed by the generic TM.

In terms of absolute performance, there are several elements that have to be mentioned to fairly analyze these first results:

- The baseline SQM is in no point an optimal SQM adapted to the generic TM or the ICAO GPS L1 C/A TM. It is just a representation of a SQM based on an achievable (but complex) receiver settings for a reference station.

- Some of the values taken in the simulation might seem pessimistic (effect of smoothing, worst case $\mathrm{C} / \mathrm{N}_{0}, \mathrm{SBAS} S Q M$ architecture), while some others might be optimistic (effect of multipath, knowledge of the reference nominal correlation function)

Figure 2 can globally be considered as representative of a minimum SQM performance with respect to a true implemented SQM in operational conditions. It is expected that more advanced metrics and SBAS architecture could allow for a safe detection of all the distortion. This is part of a separate study.

Figure 2 also shows that the ICAO-like TM can reach high worst case differential biases for undetected distortions with a MUDE above 3 meters. The analysis of the worst cases show that the 3 green circles located around values of $\overline{F O M}_{\text {max }}$ of 0.8 and differential tracking error of 3 meters correspond to distortions of type TM-C.

\section{Analysis of a Reduced Generic Threat Model Parameter Space for Galileo E1C}

This section focuses mostly on the understanding of the characteristics of the distortions of the generic TM that are not detected (according to the ICAO requirements) and that are creating a differential tracking bias greater than the MERR of $1.55 \mathrm{~m}$. As mentioned before, there is a total of 769,470 distortions that are in this case among the 10,000,000 distortions generated (7.6947\%). This set of distortions will be referred to as the "problematic" set of distortions.

A dedicated analysis was made to try to understand which parameters of the generic TM are more critical, and how the TS could be simplified or reduced. Among the many analysis performed, the effect of the number of components, the occurrence rate of the frequency components, or the amplitude of the components were looked at one by one. In order to keep the size of this article reasonable, only the main conclusions of this analysis are provided here. The main conclusion is that as long as the TS allowed for a wide variety of distortions, the worst case represented in Figure 2 (the linear link between the worst-case differential error value and the $\overline{F O M}_{\text {max }}$ ) could be reached. This was observed when reducing (in a reasonable way) the number of components, the maximum value of $\mathrm{D}$ or the maximum value of the frequency of each component. Obviously, this worst case would occur more or less often depending upon the selected parameter range, but it would generally be reached. The understanding of this phenomena was that as long as enough distortions were simulated, there would be cases where distortions would create a significant error (for its observability). As a consequence, playing with the different parameters (by reducing them, sometimes significantly) would almost systematically end up in obtaining the same worst case shape as shown in Figure 2.

Based on the analysis, it seems that the parameter that is the easiest to reduce is the maximum frequency of the components (below $20 \mathrm{MHz}$ ) as it is related to the RF front-end filter. It was also seen that it is possible to strongly stimulate the SQM, at least with a much greater impact than the ICAO-like model, with:

- A small number of components, typically lower than 5

- A unique value of $D$ that would be taken as the worst case

As a consequence, the range of values of the parameters of a new reduced generic TM could be as defined in Table 5 .

Table 5. Reduced Threat Space associated to the proposed generic TM 


\begin{tabular}{|c|c|c|c|}
\hline $\boldsymbol{N}$ & $\begin{array}{c}D \text { (correlation } \\
\text { function } \\
\text { amplitude) }\end{array}$ & $\boldsymbol{\varphi}_{\boldsymbol{k}}(\mathbf{\text { rad}})$ & $\boldsymbol{f}_{\boldsymbol{k}}(\mathbf{M H z})$ \\
\hline$[3 ; 5]$ & 0.13 & {$[0 ; 2 \pi]$} & ] $0 ; 20]$ \\
\hline
\end{tabular}

\section{RESULTS FOR GPS L1 C/A}

For the sake of comparison, the same 10 million unfiltered correlation distortions used in the previous section were also tested assuming that they now affect the correlation function of a GPS L1 C/A receiver. It is however understood that because of the correlation process, a same distortion at the correlation level does not necessarily corresponds to the same distortion at the transmitted signal level. In this frame, the baseline SQM is assumed the same in terms of required performance, location of correlators and metrics used.

\section{SQM Performance for GPS L1 C/A}

Figure 3 (left, blue circles) represents the worst case differential tracking error for GPS L1 C/A for the proposed generic TM using the exact same 10,000,000 distortions used for the Galileo E1C SQM. Superimposed to this is the worst case differential tracking error for the current GPS L1 C/A ICAO TM (green circles). For comparison purpose, the corresponding Galileo E1C is presented in Figure 3 (right). It can be seen that the proposed generic TM significantly overbounds the current ICAO TM for GPS L1 C/A. The generic model thus integrates distortions that can create much worse situations than the current ICAO TM for GPS L1 C/A.
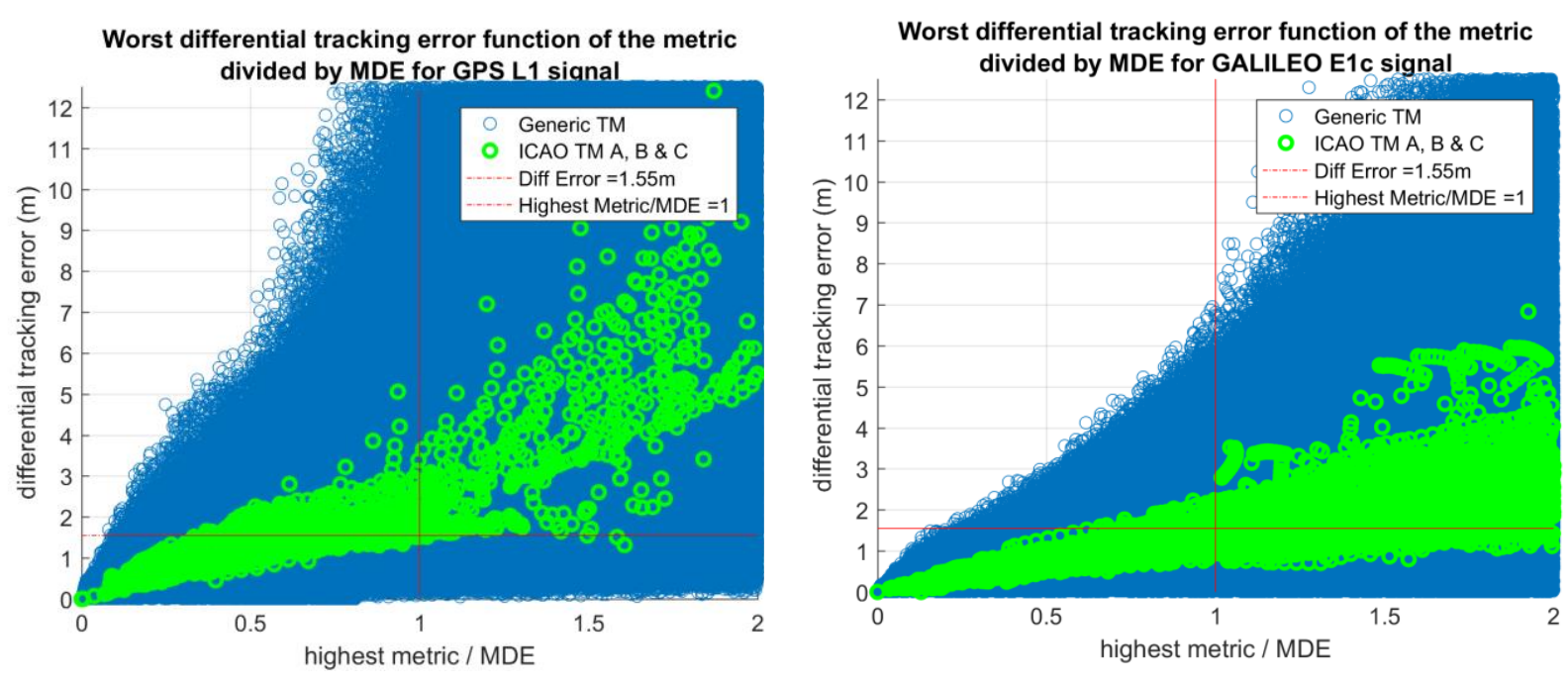

Figure 3. Worst differential tracking error as a function of the $\overline{F O M}_{\text {max }}$ at the Reference Station for GPS L1 C/A (Left) and Galileo (Right)

Figure 3 also shows that with the proposed baseline SQM, the current ICAO GPS L1 C/A TM meets the requirement of an MERR of $3.5 \mathrm{~m}$ associated to a single frequency SBAS (all distortions generated a differential tracking bias greater than $3.5 \mathrm{~m}$ is detected with the required performance). However, the new generic TM is far from providing this performance with a MUDE in excess of $15 \mathrm{~m}$. The worst case differential errors are in fact much higher than for the Galileo E1C case for the same set of distortions and the same baseline SQM. This was however expected, as the SQM performance is generally better with a sharper correlation function, as already discussed in [Pagot et al, 2016]. Generally speaking, it can be seen that the linear function bounding the worst differential tacking error as a function of $\overline{\mathrm{FOM}}_{\text {max }}$ has a slope that is about 3 times greater for GPS L1 C/A than for Galileo E1 OS. This factor 3 also corresponds to the ratio between the $\operatorname{BOC}(1,1)$ and $\operatorname{BPSK}(1)$ autocorrelation slopes.

\section{Impact of the "Problematic" Set of Galileo E1C Distortions on GPS L1 C/A SQM}

Figure 4 represents the same figure as Figure 3, except that the 769,470 distortions corresponding to the "problematic" set of distortions of Galileo E1C (see earlier section) are now highlighted in red. It can be seen that:

- many Galileo E1C "problematic" distortions can be easily detected on GPS L1 C/A 
- the Galileo E1C "problematic" distortions create worst case differential errors above 1.55m for Galileo E1C. It seems that this property is almost always preserve for GPS L1 C/A.

- the Galileo E1C "problematic" distortions do not represent well the case of low detection/low worst case differential error for GPS L1 C/A. The set of GPS L1 C/A problematic distortions would thus need to be defined separately from the one for Galileo E1 OS.

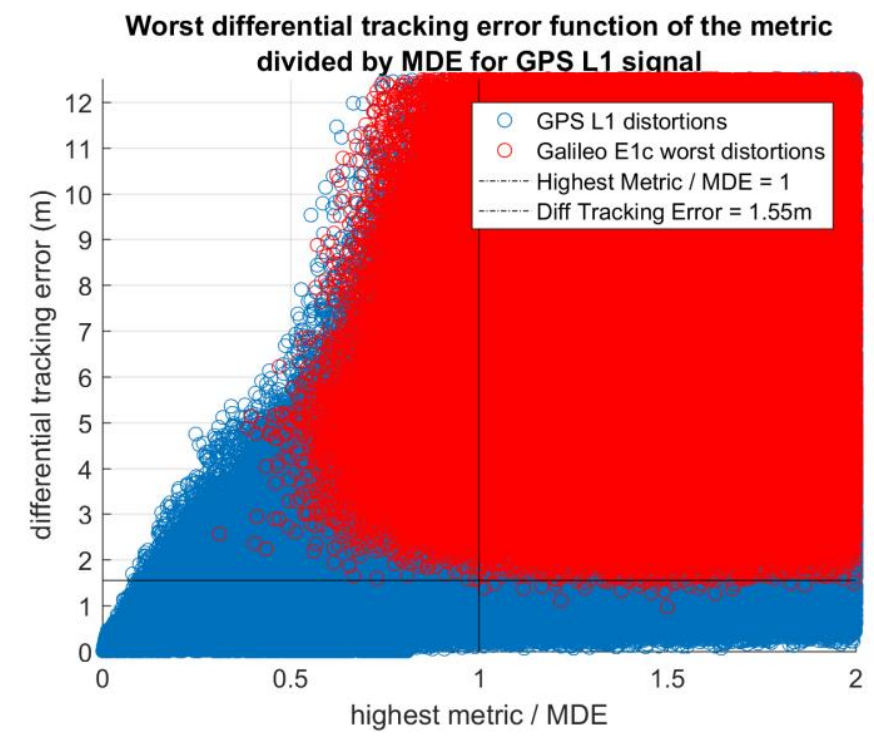

Figure 4 : Worst differential tracking error as a function of the the $\overline{F O M}_{\text {max }}$ at the Reference Station for GPS L1 C/A highlighting the Galileo E1C problematic distortions

Specific distortions were analyzed in the GPS L1 C/A and Galileo E1C case to understand this behavior. To do so, for a common correlation distortion, the impact of that distortion on the GPS L1 C/A and Galileo E1 OS correlation function and on the ratio metrics were looked at. An example is presented in Figure 5 and Figure 6 for a distortion with 4 components creating a worst case differential error of $2.99 \mathrm{~m}$ on Galileo E1C (for a $\overline{F O M}_{\max }$ of 0.51 ) and $8.93 \mathrm{~m}$ on GPS L1 C/A (for a $\overline{F O M}_{\text {max }}$ of 0.91 ). It can be seen that:

$\circ$ the effect of the same distortion is much more obvious on the GPS L1 C/A correlation function, due to its lower slope. This is the reason for resulting, in general, in a higher differential range error.

○ the MDE for the same metrics are much lower for GPS L1 C/A than for Galileo E1C. This is due to the fact that the correlation between close correlators is greater in the case of GPS L1 C/A the correlation between 2 correlators separated by $\mathrm{x}$ chips is more or less the value of the correlation function at $\mathrm{x}$ chips). This explains a better detection capability of GPS L1 C/A SQM over Galileo E1C for a given distortion.
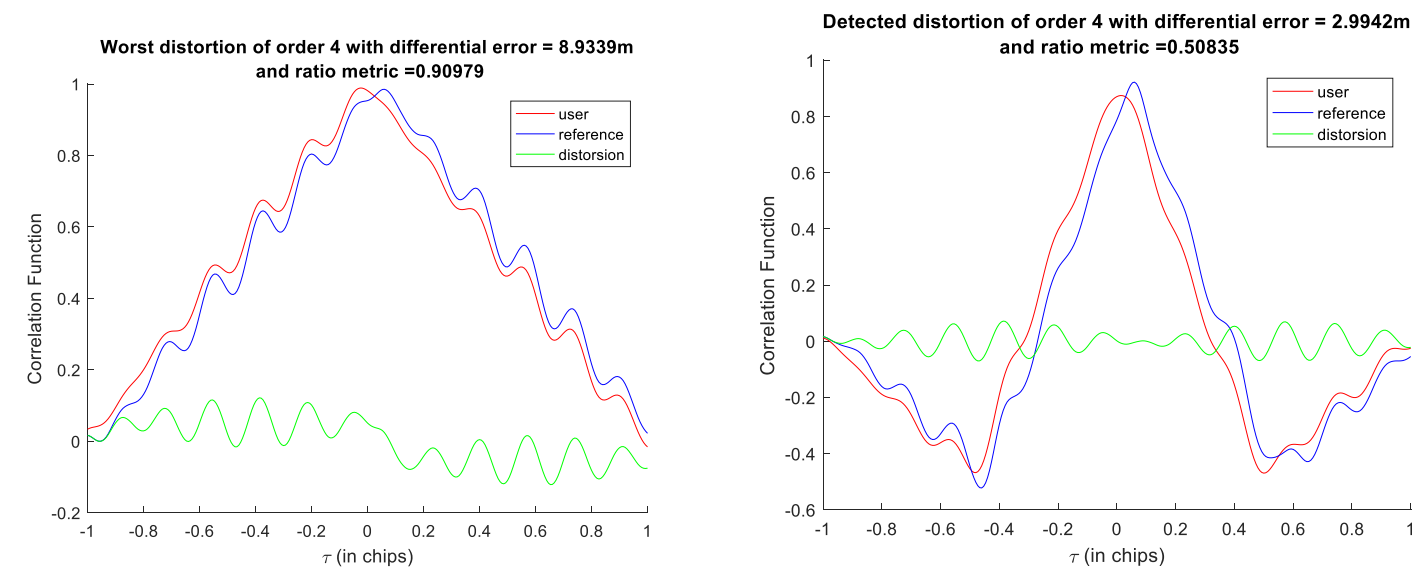

Figure 5. Distorted correlation function at reference and user receiver for a distortion (Left: GPS L1 C/A, Right: Galileo E1C) 

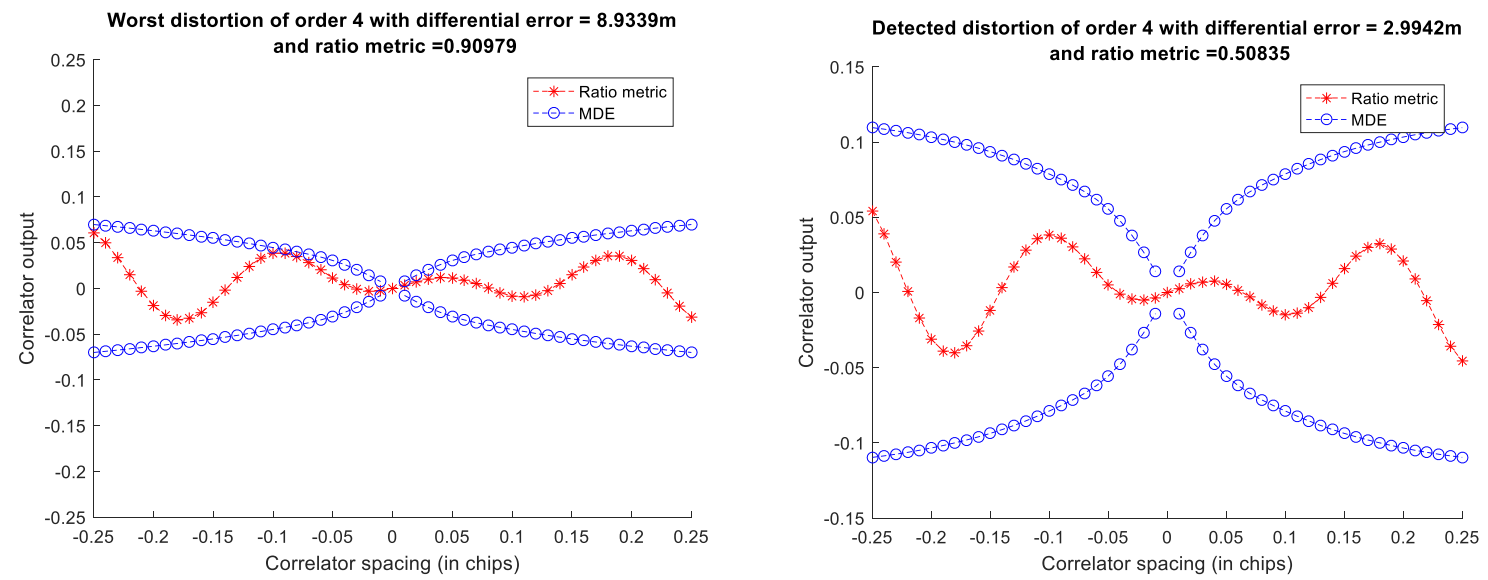

Figure 6. Ratio metric and MDE values of a distortion (Left: GPS L1 C/A, Right: Galileo E1C) - Note that the y-axis scale is different on both figures

To summarize, a same distortion will be generally easier to detect on GPS L1 C/A, however, it will generally create a larger worst case differential error (not that these are general rule of thumbs that might not systematically apply, for instance in the case where the worst user filter is not the same on the GPS L1 C/A and Galileo E1 OS). This thus explains Figure 4.

\section{POSSIBLE REDUCTION OF THE GENERIC TM}

As discussed previously, the drawback of the generic TM is that its threat space is infinite and does not provide a simple mean for testing. As a consequence, it would be important to find a way to drastically reduce its threat space, while still protecting the user against errors not represented by the current ICAO-like TM. This is the objective of this section.

\section{Initial Reduction of the Threat Space of Each Key Parameter}

It has been seen that the generic TM entails fairly large differential tracking errors compared to the ICAO-like TM, whatever the detectability of the distortion (according to the baseline SQM). This was expected as the number and nature of the distortions that are part of the generic TM are much greater than those of the ICAO-like TM. Even with a strong reduction of the threat space proposed in Table 5, the threat space of the generic TM remains extremely large. This is due to the fact that each component relies on 3 random variables (amplitude, phase and frequency). As a consequence, two drastic means for reducing the generic TM threat space are investigated hereafter.

\section{Drastic Reduction of the Threat Space of Each Key Parameter}

To further reduce the number of possible distortions to test, two decisions were made: to only take into considerations distortions with 3 components, and to roughly sample the parameter space, as shown in Table 6. The sampling of the parameters has been chosen only on the basis that it leads to a reasonably small number of distortions. As a consequence, although the sampling is very rough, the total number of distortions that can be generated based on Table 6 is equal to 13,824,000. This is higher than the 10 million distortions used before, however, it now span the entire threat space.

Table 6. Drastically Reduced Threat Space associated to the proposed generic TM

\begin{tabular}{|c|c|c|c|}
\hline $\boldsymbol{N}$ & $\begin{array}{c}\boldsymbol{D} \text { (correlation function } \\
\text { amplitude) }\end{array}$ & $\boldsymbol{\varphi}_{\boldsymbol{k}}(\mathbf{r a d})$ & $\boldsymbol{f}_{\boldsymbol{k}}(\mathbf{M H z})$ \\
\hline 3 & $\begin{array}{c}0.13 \text { (individual } \\
\text { amplitudes of each } \\
\text { component can take } \\
\text { values 0.2:0.2:1 before } \\
\text { normalization) }\end{array}$ & $1: 1: 6$ & $1: 2: 15$ \\
\hline
\end{tabular}

The generic TM with the drastically reduced threat space (all 13,824,000 distortions) has been tested for Galileo E1C with the baseline SQM. The result is presented in Figure 7 (Left). For comparison purpose, the figure obtained with the 10,000,000 
distortions used until now is provided in Figure 7 (Right). It can be seen that even with the drastically reduced threat space, the actual worst case differential tracking error for the same $\overline{F O M}_{\text {max }}$ is fairly similar to the original 10 million distortions. It can thus be noted that the MUDE is on the same order. It can also be noted that the generic TM with the drastically reduced threat space creates smaller differential tracking errors for low values of $\overline{F O M}_{\text {max }}$.
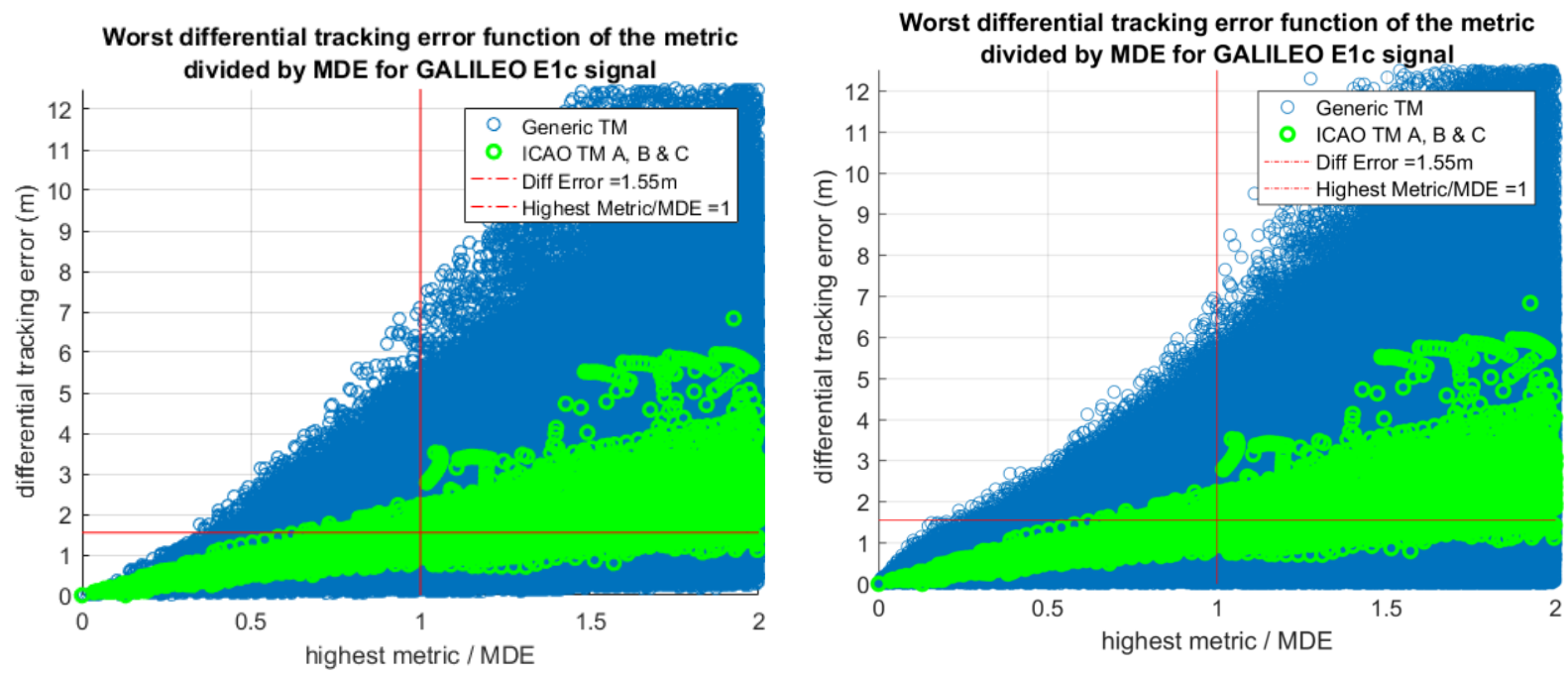

Figure 7. Worst differential tracking error as a function of the $\overline{F O M}_{\max }$ at the Reference Station for Galileo E1C with the Drastically Reduced Threat Space (Left) and the Distortion Set Used Until Now (Right)

As a conclusion, although this drastic reduction appears very rough, it still allow to have a series of distortions that create difficulties for the SQM. Its drawback however is that:

- it only represents a type of distortion (only 3 components), and is thus fairly away from the original intent of the generic $\mathrm{TM}$, which is to represent a large range of potential distortions,

- the TS is still quite large (although a"problematic" subset could be extracted)

\section{Selection of a Finite Set of Distortions}

Another approach to reduce the number of distortions from the generic TM to test is to select a number of distortions within the Galileo E1C "problematic" set of distortions. It is possible to extract a finite set of distortions that have different properties such as: easy to difficult detectability, small to large worst case differential errors, variety of number of components and frequencies. Based on the set of 769,470 problematic distortion for Galileo E1C, it should thus be easy to define a way to do that so that the characteristics of the distortion set are diverse (to avoid a set that is too dependent upon the SQM used to select the set). One way of reducing the set of problematic distortions was to select specific distortions that had specific properties, in particular, the property of testing the limits of the SQM. The goal of this section is to investigate this possibility.

Let us assume, as an example, that the set of distortions of interest would be common to all signals in the L1 band, meaning GPS L1 C/A and Galileo E1C signal. This means that it would be a common set that would be used to test the GPS L1 C/A and Galileo E1 OS SQM. As it was seen earlier, the worst distortions for GPS L1 C/A are not necessarily the worst distortions for Galileo E1 OS, and vice-versa. As a consequence, a specific mean of selection of a set of 1000 distortions (which seems like a reasonable number for testing purposes) has been put in place:

- Isolate the common undetected (according to ICAO requirements) distortions for both GPS L1 and Galileo E1 signals.

- Extract 1001 distortions based on at least one of the following criterions:

$\circ$ The induced differential error on GPS L1 is close to the Galileo E1c one (maximum difference of $1 \mathrm{~m}$ )

- The highest metric to MDE ratios on GPS L1 and Galileo E1c are close (with 0.02 maximum difference)

- The highest metric to MDE ratios is higher than 0.99 for GPS L1 and Galileo E1c

- Few additional distortions with less restrictive criterion have been add to cover the whole undetected space.

The 1001 selected distortions and the associated SQM performance for GPS L1 C/A and Galileo E1 OS are represented in Figure 8. The figure reads as follows: 
It can be seen that the selected distortions do not necessarily create high worst case differential errors on Galileo E1C, but can be hard to detect. These same distortions generally create higher worst case differential error, and are also closer to detection. This is in line with the description of the effect of a same distortion on GPS L1 C/A and Galileo E1C given earlier.

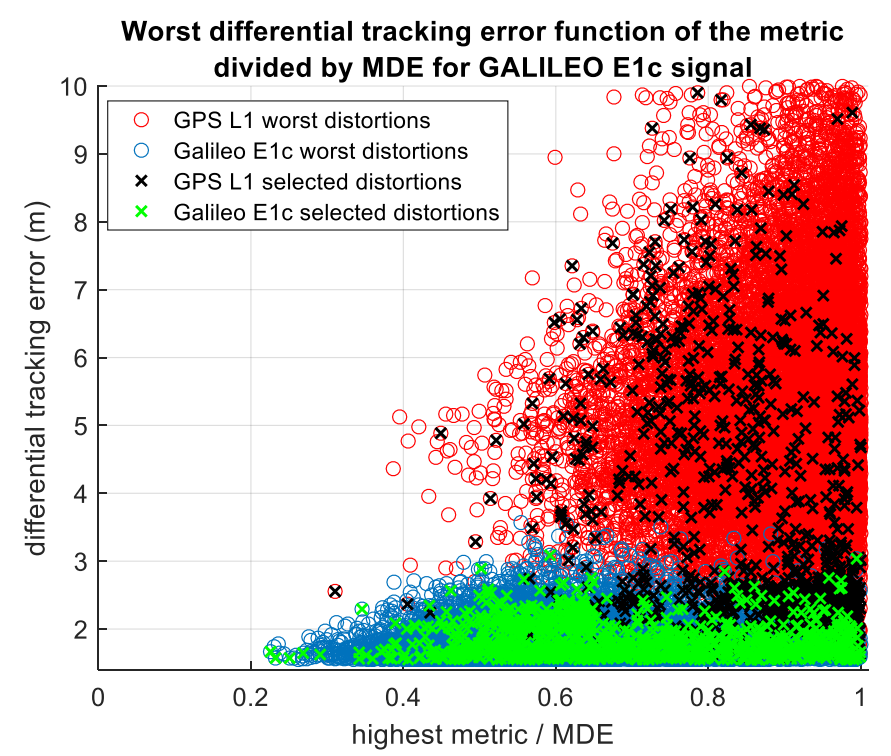

Figure 8. Selected set of distortions and Baseline SQM Performance

Figure 9 represents the distribution of the number of components and the distribution of the frequency of these components in the selected set of 1001 distortions. It can be seen that all numbers of components are well represented with an emphasis on the once with a large number of components, which seems normal as they have more degrees of freedom to create "complicated" distortions. It can also be seen that all frequency components are fairly uniformly distributed. As a consequence, this distortion set seems to represent a fairly diverse set of distortions.
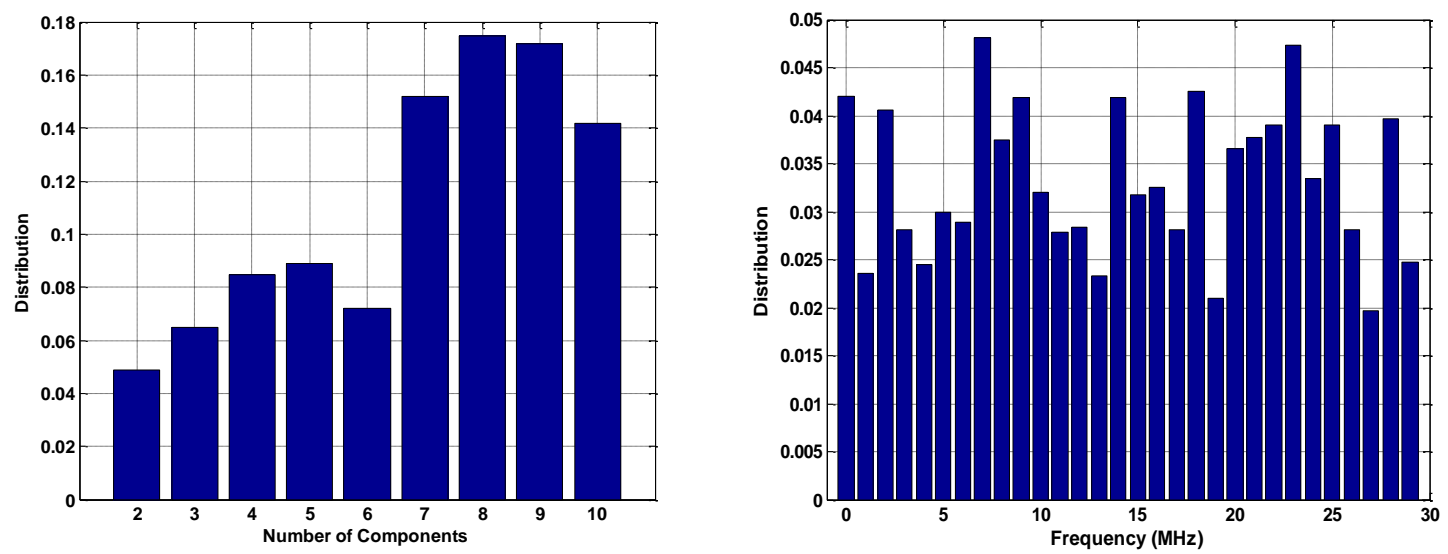

Figure 9. Distribution of the Number of Components (Left) and Frequency (Right) for the Selected Set of Distortions

Finally, it is important to understand that the set of 1001 selected distortions, although fairly diverse, has its roots in the weakness of the SQM used to define the problematic distortions. As a consequence, it is important to test a wider set of distortions (potentially the whole 10,000,000 distortions) with more advanced SQM before really deciding if this selected set is of interest. Finally, this selected set of 1001 distortions could be used together with the ICAO-like model for testing purposes. 


\section{CONCLUSIONS AND WAY FORWARD}

\section{Conclusions}

The objective of this paper was to analyze a possible new TM that could allow capturing some existing uncertainties regarding the current types of TM used by ICAO for GPS L1 C/A. Because the types of payload failure and their effects on the transmitted signal are very hard to predict, and difficult to observe as they are such rare events, the starting point was then to define a broad generic TM. This broad generic TM was then reduced essentially by taking into account some receiver considerations (maximum frequency), payload considerations (maximum power of the distortion) and its effect on a baseline SQM assumed to be achievable for future DFMC SBAS reference stations, and by trying to have a reasonable size of the TM. In particular, sections of the TM that could be detected by the baseline SQM were considered as not necessary. The TM reduction methodology thus has to be understood as a first step, since it is for now dependent upon the choice of the baseline SQM.

The investigated generic TM and its reduced versions were seen to create larger differential tracking errors than the current GPS L1 C/A ICAO TM, or than the proposed [Pagot et al, 2016] ICAO-like Galileo E1C TM, thus confirming that they represent distortions that can be more harmful than the current GPS L1 C/A ICAO TM. Such type of generic model could then be used:

- As a TM for Galileo E1 OS signal or any other new signal used in DFMC SBAS or GBAS

- $\quad$ To test an SBAS SQM function without being part of the ICAO TM

The analysis showed that some SQM optimization is still necessary to make the SQM fully adapted to protect the airborne user. This is not the case with the baseline SQM. However, to do so a certain number of further studies have to be performed and are listed in the next sub-section.

\section{Way Forward and On-Going Tasks}

The way forward will consist in several aspects:

- Consolidation of the value of the MERR for DFMC SBAS, most notably by using the concept of time-varying MERR [Rife and Phelts (2011), Phelts et al (2013)] that also includes the notion of convergence time of the smoothing process after the occurrence of an EWF and the notion of TTA. This will have an impact on the way to better select the set of distortions that could be part of the final TM.

- Improve the metric standard deviation model by analysis a set of real data coming from a reference station. This is necessary to improve the assessment of the SQM performance. This will also allow to better assess the impact of the metric smoothing process in presence of multipath.

- Think about an SBAS SQM architecture that would allow to better detect EWF, such as the centralized architecture discussed in the article

- Improve the SQM performance by finding new metrics with better performance. It was for instance observed that

$\circ$ metrics looking at the slope of the distortion, or metrics made out of a linear combination of simple ratio metrics could be interesting.

- metrics based on PRN chip observation seem to be quite promising at presented in [Thevenon et al, 2014; Phelts et al, 2013].

- $\quad$ Rationalize the SQM. This might sounds contradictory with the above bullet, but it was noted that over the 100 metrics used by the baseline SQM, some were only redundant, or at least not as critical as others. This would also be very important to make the SQM less complex.

\section{NOTE}

This paper reflects on-going R\&D of a potential approach for EWF-monitoring in SBAS. This paper should not be understood as a consolidated approach for EWF-monitoring in EGNOS.

\section{REFERENCES}

[ESA, 2016] Samson, J., Assumptions on the DFMC SBAS MOPS

[Fontanella et al, 2014] Fontanella D., Paonni M., Eissfeller B., "A Novel Evil Waveforms Threat Model for New Generation GNSS signals: Theoretical Analysis and Performance," Satellite Navigation Technologies and European

[ICAO SARPS, 2006] International Standards and Recommended Practices. Annex 10 to. ICAO. 6th Edition. July 2006.

[Pagot et al, 2016a]: Pagot J.-B., Thevenon P., Julien O., Amarillo Fernandez F., Maillard D., "Signal Quality Monitoring for New GNSS Signals", Proceedings of ION GNSS+ 2016 Conference 
[Pagot et al, 2016b]: Pagot J.-B., Thevenon P., Julien O., Amarillo Fernandez F., Maillard D., “Threat Model Design for New GNSS Signals", Proceedings of ION ITM 2016 Conference

[Phelts, 2001] R.E. Phelts, Multicorrelator Techniques for Robust Mitigation of Threats to GPS Signal Quality, Ph.D. Thesis, 2001, Stanford University, Stanford, CA

[Phelts et al, 2006] R.E. Phelts, D.M. Akos, Effects of Signal Deformations on Modernized GNSS Signals, Journal of Global Positioning Systems, Vol. 5 No. 1-2, Hong Kong, China, 2006.

[Phelts, 2013], R. E. Phelts, G. Wong, T. Walter and P. Enge, Signal Deformation Monitoring for Dual-Frequency WAAS, Proceedings of ION ITM 2013

[Rife and Phelts, 2008] J. Rife and R. E. Phelts, Formulation of a Time-Varying Maximum Allowable Error for Ground-Based Augmentation Systems, IEEE Transactions on Aerospace and Electronic Systems, Vol 44, No 2, April 2008

[Schloss et al, 2002] P. Schloss, R. E. Phelts, T. Walter, P. Enge, A Simple Method of Signal Quality Monitoring for WAAS LNAV/VNAV, Proceedings of ION NTM 2002

[Shively, 1999] Shively, C. A., "Derivation of Acceptable Error Limits For Satellite Signal Faults in LAAS," ION GPS-99.

Proceedings of ION GPS 1999. 